REVESCO. Revista de Estudios Cooperativos

ISSN: $1885-8031$

\title{
La Empresa Social: realidad de la Economía Social en España
}

\author{
Macarena Pérez-Suárez ${ }^{1}$, Isadora Sánchez-Torné ${ }^{-}$y Daniel Pérez-Troncoso ${ }^{3}$
}

Recibido: 12 de marzo de 2021 / Aceptado: 28 de junio de 2021 / Publicado: 22 de octubre de 2021

Resumen. El objetivo de la investigación fue identificar el actual tejido socio empresarial de España, así como detallar el perfil de la Empresa Social activa. En esta identificación, se planteó una investigación inductiva mediante un análisis cuantitativo a partir de fuentes secundarias, en particular, desde cuatro directorios públicos de Empresas Sociales localizadas en España. A partir de los cuales se elaboró una base de datos (directorio) propia con varias variables comunes (tamaño, sector, año de constitución, región) sobre las características corporativas. Cuando se obtuvieron los datos, se trataron con Stata, en un análisis estadístico descriptivo e inferencial de una población localizada de 2.587 empresas. Se determinaron varias características definitorias de las actuales Empresas Sociales de España, como la concentración territorial (la mayoría se ubica en tres regiones: Andalucía, Comunidad de Madrid y Cataluña) donde arraiga la Economía Social. Este perfil conectó en la conceptualización nacional de Empresa Social, al contribuir a la literatura dando respuesta a las apelaciones originales de diversas autorías para contrastar empíricamente las deducciones existentes de la organización social con la variedad de experiencias y modelos. Así, este estudio se situó en una línea de investigación emergente de mapeo y tipificación territorial.

Palabras clave: Empresa Social; Ciclo de vida de la organización; Cooperativa de Iniciativa Social; Economía Social; España.

Claves Econlit: B55; L30; P13.

\section{[en] Social Enterprise: a reality of the Social Economy in Spain}

\begin{abstract}
The objective of the research was to identify the current social business fabric in Spain, as well as to detail the profile of the active Social Enterprise. In this identification, an inductive investigation was proposed through a quantitative analysis from secondary sources, in particular, from four public directories of Social Enterprises located in Spain. From which a self database (directory) was prepared with several common variables (size, sector, year of incorporation, region) about corporate characteristics. When the data were obtained, they were processed with Stata, in a descriptive and inferential statistical analysis of a localized population of 2,587 companies. Several defining characteristics of the current Social Enterprises of Spain were determined, such as territorial concentration (most are located in three regions: Andalusia, Community of Madrid and Catalonia) where the Social Economy takes root traditionally. This profile of Social Enterprises derived from the study contributed to the national literature by responding to the theories of various authors and empirically confirming the existing deductions about social organization with the variety of experiences and models obtained. Therefore, this study was placed in an emerging research line of mapping and typification territorial.
\end{abstract}

Keywords: Social Enterprise; Organizational Life Cycle; Social Initiative Co-operative; Social Economy; Spain.

Sumario. 1. Introducción. 2. Revisión de la Literatura. 3. Metodología. 4. Resultados. 5. Conclusiones. 6. Referencias bibliográficas.

Cómo citar. Pérez-Suárez, M.; Sánchez-Torné, I.; Pérez-Troncoso, D. (2021) La Empresa Social: realidad de la Economía Social en España. REVESCO. Revista de Estudios Cooperativos, vol. 139, e77445. https://dx.doi.org/10.5209/reve.77445.

\section{Introducción}

La Empresa Social es una realidad socioeconómica ascendente en los últimos años, por tanto, durante una crisis económica. La experiencia europea es significativa y, hoy, las instituciones trabajan en un marco común para la amplísima diversidad de realidades empresariales, e introducir una etiqueta de Empresa Social Europea (Altzelai, 2020). A nivel territorial, se reconoce que hay mayor número de Empresas Sociales en

1 Universidad de Sevilla, España.

Dirección de correo electrónico: mperez32@us.es

2 Universidad de Sevilla, España.

Dirección de correo electrónico: isanchez6@us.es.

3 Universidad de Granada, España.

Dirección de correo electrónico: danielperez@go.ugr.es 
Reino Unido, por su veterano emprendimiento y en Francia, por su atención a la ayuda social. No obstante, la Comisión Europea impulsa a estas iniciativas ${ }^{4}$ como demuestran la Social Business Iniciative (SBI) ${ }^{5}$ y el Programa de la Unión Europea para el Empleo y la Innovación Social 2014-2020 (EaSI) ${ }^{6}$ a favor de un ecosistema europeo común en emprendimiento social. El concepto de Empresa Social es "de amplia tradición en Europa y desarrollado durante muchos años bajo diferentes formas jurídicas, al que se pueden incorporar elementos novedosos (...) dentro de las nuevas propuestas de empresas comprometidas con el desarrollo humano" (Fisac-García, Moreno-Romero, Mataix y Palacios, 2011:14). En este momento, se expresan los ecosistemas (Arabadzhieva y Vutsova, 2021) y los significados locales de la Empresa Social (Pfeilstetter y Gómez-Carrasco, 2020).

En España, "los orígenes de las empresas sociales están en la solidaridad y en los valores colectivistas de las organizaciones asociativas, mutualistas y cooperativas" (Comisión Europea, 2020:42) aunque no destaca el emprendimiento social ${ }^{7}$ (GEM, 2014:118) ${ }^{8}$. Según datos publicados por la entidad Cáritas, estas entidades sociales obtuvieron una facturación en su conjunto de 37 millones de euros con una inversión en acciones de inserción laboral de 27 millones de euros en 2016 ${ }^{9}$. Sin embargo, el país no dispone de fuentes de información fiables sobre este tejido empresarial, y si existe un consenso sobre que las empresas españolas están muy centradas en ayudar a colectivos en riesgo de exclusión ${ }^{10}$.

La vinculación entre la Empresa Social y la Economía Social es reconocida (Caro-González, PérezSuárez y Sánchez-Torné, 2019), pues "una Empresa Social, agente de la Economía Social, es una empresa cuyo principal objetivo es tener una incidencia social" (Monzón-Campos y Chaves-Ávila, 2012:21). Desde ahí, las autorías puntualizan: ellas operan "en el mercado proporcionando bienes y servicios de manera innovadora y utiliza sus excedentes para fines sociales" (Fajardo, 2014:79); "persiguen una misión social y tienen una lógica económica" (Berbegal-Mirabent, Mas-Machuca y Guix, 2021) y "hacen posibles los objetivos marcados en la Estrategia 2020 de la Unión Europea" (Díaz-Foncea, Marcuello y MarcuelloServós, 2012:181). En definitiva, tal como identificó la Comisión Europea (2011), una empresa social ${ }^{11}$ es "un operador cuyo objetivo principal es tener un impacto social (...) utiliza sus beneficios principalmente para lograr objetivos sociales. Se gestiona de forma abierta y responsable, implicando en particular a los trabajadores, consumidores y grupos de interés afectados por su actividad comercial". Aun así, "la mayoría de las empresas sociales europeas (...) reúne a todas aquellas organizaciones cuyo objetivo principal es servir a sus socios o una comunidad en lugar de buscar la maximización de la rentabilidad económica" (Nogales-Muriel, 2017:120).

El concepto de Empresa Social establecido por la escuela europea EMES se usa dentro de la literatura académica (Díaz-Foncea y Marcuello, 2014) y tiene reconocimiento institucional (Monzón-Campos y Chaves-Ávila, 2012), pues "el enfoque EMES conforma el concepto de Empresa Social que ha prevalecido en las instituciones de la Unión Europea" (Caro-González, et al., 2019:131). La definición de Empresa Social de EMES $^{12}$ (European Research Network ${ }^{13}$ ) identifica once criterios agrupados en tres dimensiones: económica y emprendedora, social y gobernanza participativa (Defourny y Nyssens, 2014). Entendiendo a estas empresas como "aquellas organizaciones privadas no lucrativas que proporcionan bienes y servicios directamente relacionados con su objetivo explícito de beneficio a la comunidad" (Díaz-Foncea y Marcuello, 2014:146). "La ausencia de fin lucrativo es una protección frente a dos fenómenos negativos: la selección adversa y el riesgo moral" (Laville, 2015:94). Para Fisac-García, et al. (2011:5), la organización "se apoya en dinámicas colectivas que incluyen diferentes tipos de grupos de interés en sus órganos de gobierno, tienen una fuerte autonomía y soportan riesgos económicos asociados a su actividad". Aunque reconocen que existe una ambigüedad en el concepto dada la diferencia de enfoques académicos, la falta de un marco teórico común, y la frecuencia con la que el término Empresa Social procede de la traducción de distintos términos no equivalentes (Fisac-García, et al., 2011:4). La definición es complementada por otros elementos como el

Pérez de Pablos, S. (2013). (Consultado 15.01.2021). https://elpais.com/sociedad/2013/02/24/actualidad/1361743315 132277.html.

Comisión Europea. (Consultado 25.01.2021). https://ec.europa.eu/growth/sectors/social-economy/enterprises en.

Comisión Europea. (Consultado 25.01.2021). https://ec.europa.eu/social/main.jsp?catId=1081\&langId=es.

España presenta una mínima tasa de actividad emprendedora social (un 0,51\% frente al 4,25\% de Islandia, por ejemplo). APD. 'Informe Forética 2015 sobre el estado de la Responsabilidad Social Empresarial en España'. (Consultado 15.01.2021). https://www.apd.es/la-importancia-delemprendimiento-social-espana/.

8 Global Entrepreneurship Monitor. 'Informe GEM España'. (Consultado 01.02.2021). http://www.gem-spain.com/wpcontent/uploads/2015/04/GEM es 2014.pdf.

Cáritas Trabajo. (Consultado 25.01.2021). http://www.alfayomega.es/107937/caritas-cuenta-ya-con-49-empresas-de-economia-social-en-espana.

10 Personas discapacitadas, personas enfermas mentales, personas ex drogodependientes, personas expresidiarias, mujeres maltratadas, personas paradas de larga duración o jóvenes con formación escasa.

11 COM/2011/0682 de 25 de octubre de 2011 (Consultado 15.01.2021). https://eur-lex.europa.eu/LexUriServ/LexUriServ.do?uri=COM:2011:0682:FIN:ES:PDF.

12 La red europea EMES-(EMES es el acrónimo en francés de un gran amplio proyecto de investigación llevado a cabo sobre la "Emergencia de las Empresas Sociales en Europa" (1996-1999). Originalmente se refería al grupo de investigadores que desarrollaron este proyecto para la Dirección General de Investigación de la Comunidad Europea. El nombre se mantuvo en los sucesivos proyectos y en la actualidad, la red EMES está compuesta por nueve centros de investigación especializados en estos temas, así como otros investigadores particulares de toda Europa)dedicada al estudio de las empresas sociales (Fisac-García, et al., 2011:5).

13 Red europea EMES. (Consultado 15.01.2021). https://emes.net/. 
ecosistema colaborativo, la sostenibilidad económica e impacto social (Fisac-García y Moreno-Romero, 2015).

Este significado ya fue distinguido por Argudo (2011:180), al mostrar que las empresas sociales se caracterizan "por su finalidad social, su carácter no lucrativo y su gestión de carácter empresarial". Su acción "genera cohesión social y promueve la solidaridad, pero al mismo tiempo se la desplaza de aquellos espacios más rentables" (Salinas y Rubio, 2001:102). Según Díaz-Foncea, Marcuello y Marcuello-Servós (2012:183), "la definición de Empresa Social requiere de una aproximación multidimensional en la que se conjugan elementos tradicionales de la empresa y de la Economía Social, que afectan especialmente al objeto social, la motivación económica y los modelos de gestión”. Finalmente, Solórzano-García, Guzmán-Alfonso, SavallMorera y Villajos-Girona (2018:160), matizan que las empresas sociales tienen como objetivo "la generación de valor social para los beneficiarios de su misión social y su fuente de ingresos principal es la vía comercial (...) como medio para obtener sus fines". Se hallaría una convivencia entre las empresas sociales creadas como unas entidades de inserción por organizaciones no lucrativas y las empresas sociales creadas como una sociedad limitada o cooperativa.

La conceptualización explícita evidencia un tratamiento académico en auge. La academia aborda una aparición y una consolidación plurales de la Empresa Social en disímiles contextos y modelos organizacionales. Si bien el escenario nacional se inclina por un tratamiento conceptual en detrimento del tratamiento empírico al revelar diferentes modelos en función de su procedencia y ciertas diferencias con otras tradiciones europeas (Spear, Cornforth y Aiken, 2009; Díaz-Foncea, Marcuello y Marcuello-Servós, 2012; Díaz-Foncea y Marcuello, 2014).

Determinado el contexto, el objetivo de la investigación es identificar el actual tejido socio empresarial de España. Con tal fin, se plantea un estudio exploratorio inicial estructurado en cuatro partes, desde limitar la revisión de la literatura hasta referir varias deducciones. Esta investigación trata una realidad específica y singular que proporciona información e insta a afanar unas exploraciones más ambiciosas, así la principal aportación científica es discutir algunos tópicos del fenómeno estudiado y generar conocimiento para la comunidad (la Administración Pública, las personas del emprendimiento social, las personas de la Economía Social, etc.) que contribuyen tanto a la sostenibilidad futura de las entidades sociales como a su integración real en la Economía Social.

\section{Revisión de la Literatura}

El origen de la Empresa Social de España se data en el siglo XV (Solórzano-García et al., 2018). Tal como ya detalló Nogales-Muriel (2017:130), "en España, la Empresa Social no aparece en los últimos años de modo aislado, en el vacío. De hecho, tal desarrollo se debe en gran medida al excepcional caldo de cultivo que supone la presencia de la Economía Social".

Las investigaciones desarrolladas en España sobre las empresas sociales identifican tres líneas de trabajos: el transcurso de la organización no lucrativa de acción social a la empresa social (Salinas y Rubio, 2001; Argudo, 2011; Fisac-García, et al., 2011); la identificación de los modelos organizativos nacionales (Díaz-Foncea y Marcuello, 2014; Nogales-Muriel, 2017); y la delimitación socioeconómica de la Empresa Social (Solórzano-García et al., 2018). Tres líneas de trabajo teóricas que hallan su excepción en los trabajos empíricos sobre las empresas de inserción (EI) (Paniagua, 2013; Retolaza, San-José y Araujo, 2014; Nogales-Muriel, 2017; Martínez-Rueda, Aróstegui y Galarreta, 2018; García, 2020; García, Herrero y Martínez, 2020).

A nivel teórico, se identifican tres tipos principales de entidades sociales: las Empresas de Inserción (EI), los Centros Especiales de Empleo (CEE) y las Cooperativas de Iniciativa Social (CIS) (Díaz-Foncea, Marcuello y Marcuello-Servós, 2012; Díaz-Foncea et al., 2017; Nogales-Muriel, 2017; Solórzano-García et al., 2018). Se suman, algunas asociaciones y fundaciones (Argudo, 2011), la Sociedad Cooperativa de Impulso Empresarial y las Sociedades Cooperativas de Servicios Públicos de la Ley 14/2011 de Cooperativas Andaluzas (Nogales-Muriel, 2017).

Las Cooperativas de Iniciativa Social (CIS) pueden configurarse "como especialidad de cualquier tipo de cooperativa dentro de las clasificaciones que se contemplan legislativamente" (Art. 106 de la Ley 27/1999 de Cooperativas). La singularidad determina la calificación del ánimo social siendo obligatorio un objeto social inscripto y una serie de requisitos reflejados en los estatutos sociales («cooperativa sin ánimo de lucro») (Argudo, 2011:183). En España, "el término «cooperativa social» no es recogido expresamente en la legislación, por lo que se trata de una denominación «de conveniencia» con la intención de definir a un conjunto concreto de cooperativas" (Burgo, 2014:77). Sin embargo, la Ley 27/1999 regula dos tipos de figuras jurídicas: la cooperativa de integración social y la cooperativa de iniciativa social. Ellas se describen por "su fin mutualista (atender las necesidades y aspiraciones de sus socias y sus socios), pues cumplen una función de interés social destacable" (Fajardo, 2014:6). Hasta la fecha se evidencia que las escasas aportaciones científicas proceden del ámbito jurídico (Rodríguez, 2015), si bien ha sido señalado que "estas 
entidades cumplen todas las características del sector de la Economía Social (ya que las cooperativas constituyen la forma jurídica más representativa) con la característica adicional de estar dedicadas a servir al interés general de la sociedad. En España, éstas surgen al final del siglo XX con el propósito de crear dentro del marco nacional de Economía Social un modelo organizativo similar al de las cooperativas sociales italianas" (Solórzano-García et al., 2018:167).

La realidad de los Centros Especiales de Empleo (CEE) en la economía española es abordada como un modelo de negocio social (López, Maside y Torrelles, 2019) y un modelo normativo (Sánchez, 2020). Por su parte, los trabajos empíricos nacionales sobre las Empresas de Inserción (EI) se hallan a lo largo del quindenio reciente (Puig, 1998; Álvarez, 1999; Coque y Pérez, 2000; Rojo, 2000; López-Aranguren, 2002; FAEDEI, 2003; Millán, 2006; García y Esteve, 2007; Marcuello, 2007; Quintão, 2007; Marcuello, Bellostas y Marcuello-Servós, 2008; Marcuello y Marcuello-Servós, 2010; Melián, Campos y Sanchis, 2011; Paniagua, 2013). En la práctica, la EI tiende a adoptar una diversidad de figuras jurídicas, predominando las asociaciones y fundaciones frente a las sociedades limitadas, pues su desarrollo incide directamente en el nivel de cohesión social y solidaridad de la sociedad ${ }^{14}$. De ahí la recomendación de replantear el modelo de inserción (Retolaza, San-José y Pruñonosa, 2013) y las nacientes medidas públicas de apoyo (Comisión Europea, 2020).

En síntesis, tal como señalan Solórzano-García et al., (2018:160), "a nivel nacional, establecer una clasificación de empresas sociales es aún una tarea pendiente" aunque se hallan algunos intentos (DíazFoncea et al., 2017; Comisión Europea, 2020). Se puede derivar que hay ciertos factores que han ralentizado el desarrollo de la Empresa Social en España como es: "el legado histórico de la distinción entre Economía Social, economía solidaria y tercer sector de acción social que ha resultado en una separación de facto entre las organizaciones" (Nogales-Muriel, 2017:132). Al mismo tiempo, sobre el estado de la literatura de la Empresas Sociales de España, se puede decir que: la investigación es limitada y de tendencia teórica, y se identifican tres modelos organizativos (Empresas de Inserción, Centros Especiales de Empleo y Cooperativas de Iniciativa Social) con características propias que "deben generar no sólo valor social, sino que también valor económico" (Torres, 2017:63).

A partir de ahí, y considerando las investigaciones previas, se formulan tres hipótesis:

$\mathrm{H}_{1}$ : Las Empresas Sociales españolas se crean en periodo de crisis económica como durante la Gran Recesión (2007-2016).

$\mathrm{H}_{2}$ : Las Empresas Sociales españolas ostentan unas características propias.

$\mathrm{H}_{3}$ : Las Empresas Sociales residen donde la Economía Social nacional.

\section{Metodología}

Los antecedentes residen en dos estimaciones conocidas: "según cifras oficiales analizadas a 2016, el número estimado total de empresas sociales en España durante 2014 asciende a 8.410" (Nogales-Muriel, 2017:125) y el universo es de 9.680 de empresas sociales en España a 2017 (Comisión Europea, 2020:115).

Por consiguiente, esta investigación analizó al tejido empresarial de las Empresas Sociales españolas en 2020. En primer lugar, se elaboró un directorio propio a partir de cuatro Websites que coinciden en la tipificación de empresa social mediante la precisión del objeto social e identificación del bien común (Cuadro $n^{\circ} 1$ ).

Cuadro. 1. Fuentes digitales consultadas.

\begin{tabular}{|c|c|c|c|}
\hline Nombre & Objetivo & $\begin{array}{c}\text { Referencia } \\
\text { territorial }\end{array}$ & Sitio Web \\
\hline Ashoka & $\begin{array}{l}\text { Componer una red global de personas } \\
\text { emprendedoras sociales. }\end{array}$ & Madrid & $\begin{array}{l}\text { https://spain.ashoka.org/emprendimien } \\
\text { to-social/emprendedores-sociales/ }\end{array}$ \\
\hline $\begin{array}{l}\text { Interès } \\
\text { Social }\end{array}$ & $\begin{array}{l}\text { Formar un espacio accesible a dar } \\
\text { cobertura a las actividades de integración } \\
\text { sociolaboral de colectivos en riesgo de } \\
\text { exclusión social. }\end{array}$ & Barcelona & $\frac{\text { http://www.interesocial.com/empresas- }}{\underline{\text { sociales/ }}}$ \\
\hline Redcreactiva & $\begin{array}{l}\text { Constituir un espacio de intercambio de } \\
\text { conocimiento y buenas prácticas en }\end{array}$ & Valencia & http://www.redcreactiva.org/directorio \\
\hline
\end{tabular}

14 Ejemplo es, tal como recogen Aretxabala y Caro (2013:180), que en Euskadi la inmensa mayoría de las EI cuenten con personas inmigrantes internacionales. 


\begin{tabular}{|c|l|c|c|}
\hline & emprendimiento social. & \\
\hline Social & $\begin{array}{l}\text { Atender la Iniciativa Social Enterprise } \\
\text { España como un ecosistema español de } \\
\text { emprendimiento social e inversión de } \\
\text { impacto e impulso a sus empresas sociales. }\end{array}$ & Madrid & $\underline{\underline{\text { https://socialenterprise.es/programas/c }}}$ \\
\hline \begin{tabular}{l} 
ategoria/empresas-sociales-espana/ \\
\hline
\end{tabular}
\end{tabular}

Fuente: Elaboración propia.

Esta labor de recopilación de información se llevó a cabo durante los meses de marzo, abril y mayo de 2020, permitiendo encontrar una muestra objeto de estudio de 2.587 empresas sociales que derivó en la consulta de cada sitio web corporativo. Tomando la población referencia de 9.680 entidades y con un nivel de confianza de $95 \%$, el error muestral es del $2 \%$. A continuación, se plateó un estudio agregado de las 2.587 corporaciones para conocer sus características específicas mediante el uso de Stata (v.15) para realizar un análisis descriptivo e inferencial.

El análisis descriptivo tuvo como objetivo identificar el actual Perfil de Empresa Social activa y agrupar el año de creación de la empresa por el mayor número de respuestas halladas para establecer el ciclo de vida de la organización (Bretos, Errasti y Marcuello, 2020; Sánchez-Torné y Pérez-Suárez, 2020). La categorización del ciclo de vida se estableció de la siguiente forma: nacimiento (1 año de vida), crecimiento ( 2 a 7 años), madurez ( 8 a 16 años) y madurez avanzada (16 años en adelante). Posteriormente, se procedió a realizar una agrupación dual: el grupo de nacimiento y crecimiento y el grupo de madurez y madurez avanzada. Por su parte, el análisis de inferencias consistió en la aplicación de la prueba $Z$ para contrastar proporciones a un nivel de confianza del $95 \%$ y el error del 5\%, con el objetivo de estudiar si existen diferencias significativas en el perfil de los dos grupos determinados.

En último lugar, hay que apuntar que los cálculos no contabilizan las respuestas $\mathrm{Ns} / \mathrm{Nc}$, y que se han tratado ocho variables: la forma jurídica, la localización (Comunidad Autónoma), el volumen de facturación, el tamaño, el servicio, el sector productivo, el periodo de constitución y el ciclo de vida.

\section{Resultados}

\subsection{El perfil de la Empresa Social activa en España}

\subsubsection{Sector de producción}

Gráfico. 1. Sector de Producción de las Empresas Sociales de España.

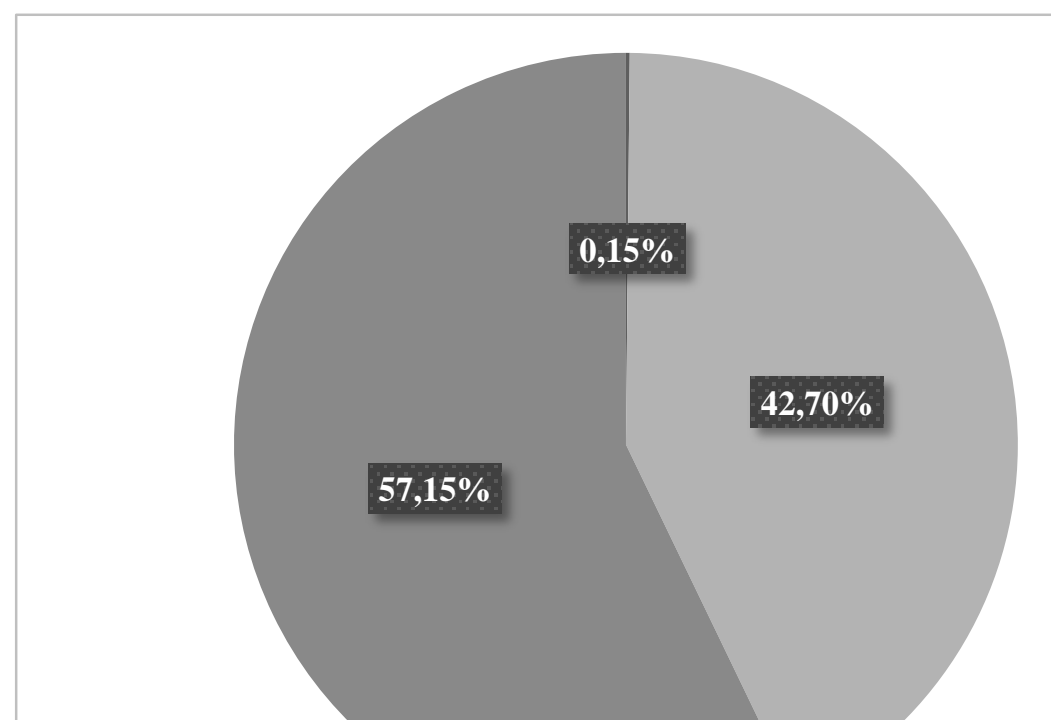

Fuente: Elaboración propia a partir de Ashoka, Redcreactiva, Social Enterprise e Interès Social (2020).

Tal como indica el Gráfico 1, las Empresas Sociales de España operan en el Sector Terciario $(57,15 \%)$ y en el Sector Secundario (42,70\%), mientras que el Sector Primario es restringido. 


\subsubsection{Servicios prestados}

En el Gráfico 2 se muestran los servicios que presentan las empresas sociales en España, la mayoría de las empresas sociales se dedica a la Sanidad (48,72\%), la Manufacturación (15,77\%) y a la Administración u otras actividades auxiliares $(13,60 \%)$.

Gráfico. 2. Servicios prestados por las Empresas Sociales de España.

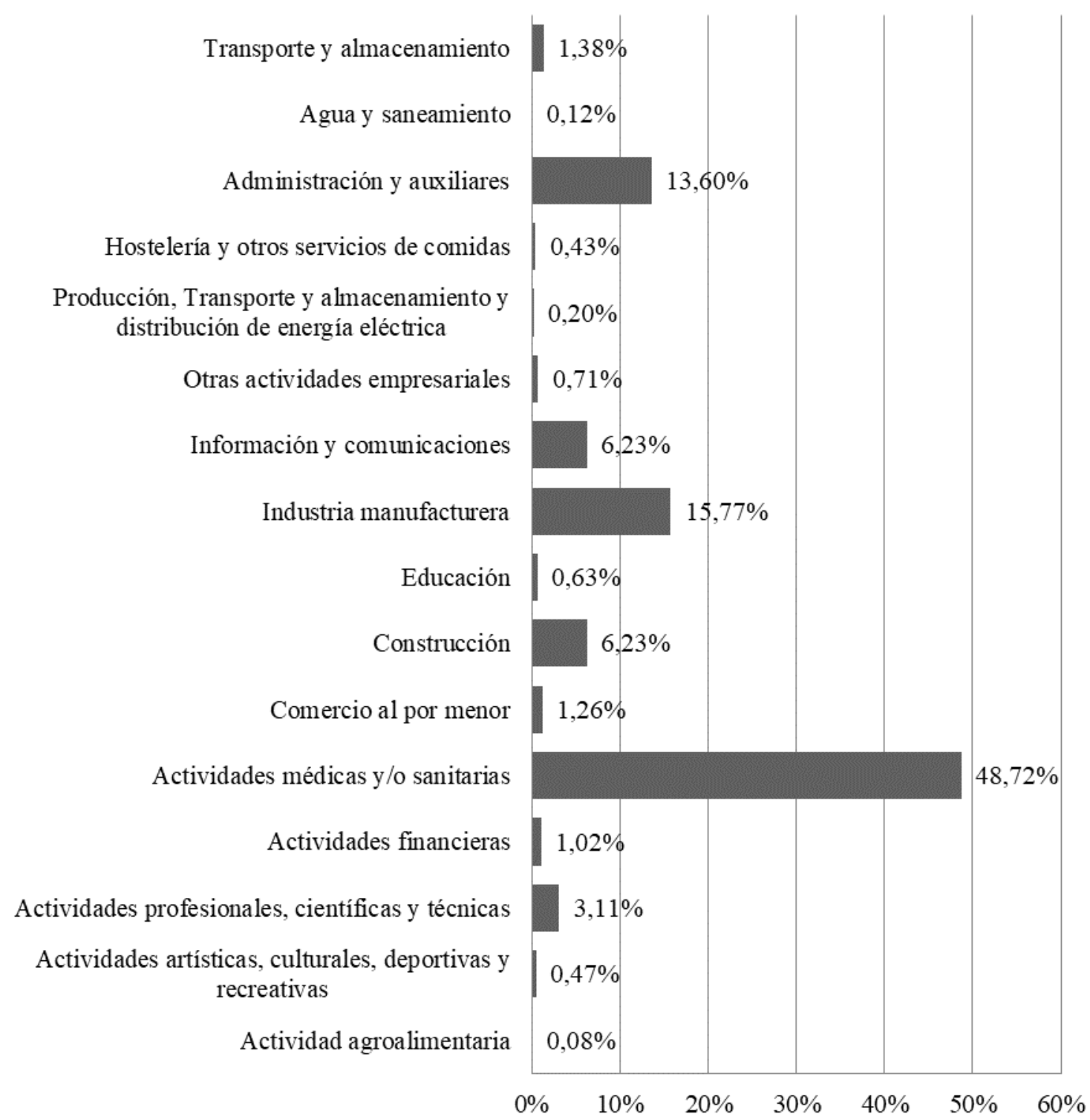

Fuente: Elaboración propia a partir de Ashoka, Redcreactiva, Social Enterprise e Interès Social (2020).

\subsubsection{Tipo de empresa}

La forma jurídica de la Empresa Social de España (Gráfico 3) es, mayoritariamente, una Sociedad Limitada (62.85\%), una Cooperativa (23.54\%) o una Sociedad Laboral (11.48\%). Un resultado que se opone a lo señalado por Aretxabala y Caro (2013), pues les otorgaba una mayoría a las fundaciones frente a las sociedades limitadas en España. Esto reafirma el auge de la Sociedad Limitada con objeto social señalado por Sánchez-Torné y Pérez-Suárez (2020). 
Gráfico. 3. Forma Jurídica de las Empresas Sociales de España.

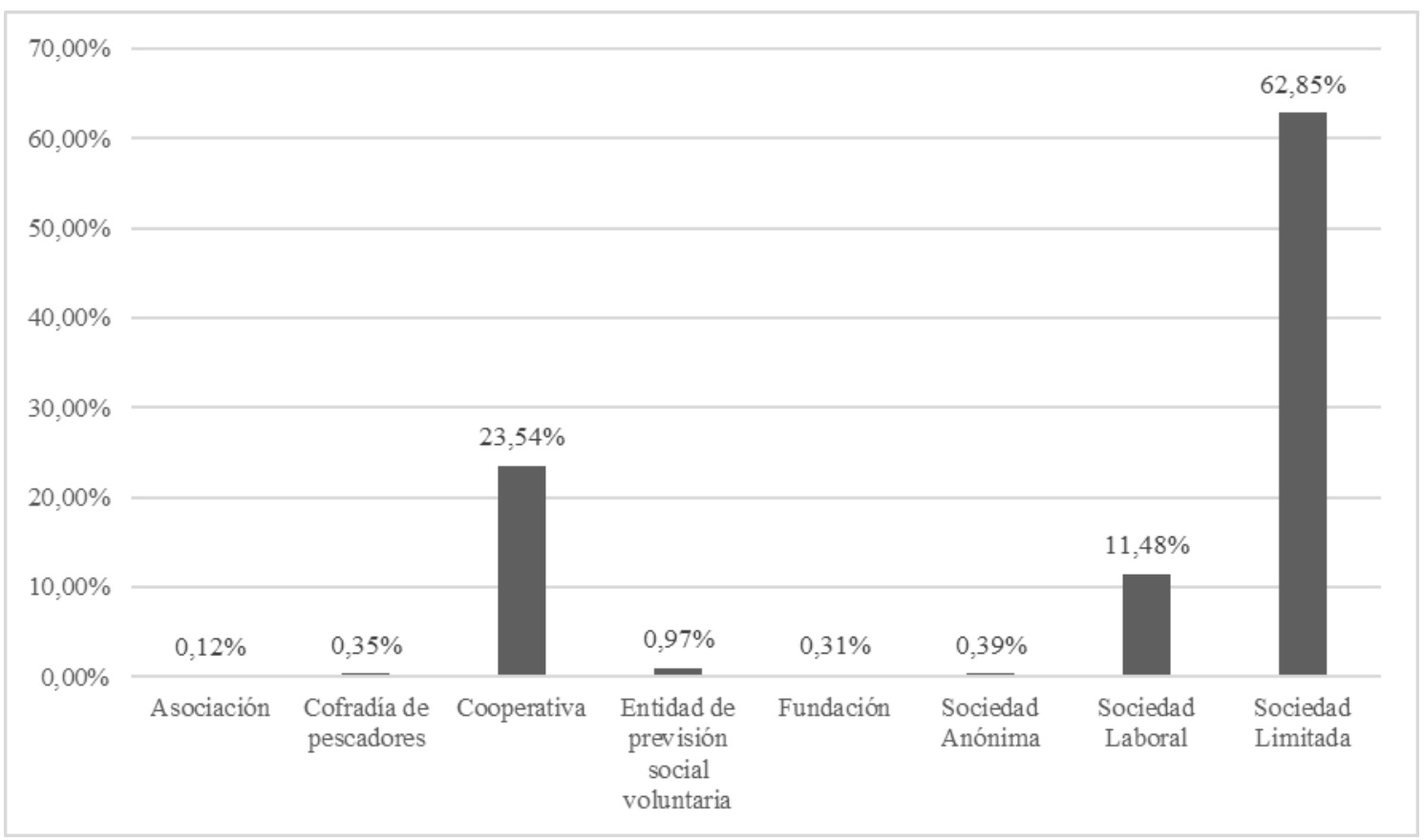

Fuente: Elaboración propia a partir de Ashoka, Redcreactiva, Social Enterprise e Interès Social (2020).

El 23,54\% cooperativo señala que son 595 sociedades activas en 2020 de las 647 cooperativas de iniciativa social (CIS) activas en 2017. Una primera observación positiva de subsistencia empresarial. No obstante, habría que comprobar si se mantiene la siguiente averiguación con datos de 2017: "las CIS están localizadas principalmente en Andalucía, Cataluña, País Vasco y Madrid. Las CIS son entidades de pequeño tamaño, que tienden a crearse con menos de 5 socios y un capital social inicial de menos de 5.000 euros. Su actividad se localiza fundamentalmente en los sectores de Educación y de Servicios sociales y sanitarios" (Bretos, Díaz-Foncea y Marcuello, 2020:1).

\subsubsection{Localización}

La mayoría de Empresas Sociales en España residen en las comunidades autónomas más pobladas (Tabla 1): 1) Andalucía $(19,95 \%)$, 2) Cataluña (13,34\%) y 3) Comunidad de Madrid (17,43). Al atender a la variable poblacional, aquellas regiones con una mayor proporción de empresas sociales por número de habitantes son Andalucía, Comunidad de Madrid, Aragón, Navarra, País Vasco y Galicia. A la cola, sin embargo, se encuentran las Islas Canarias y Baleares, y La Rioja. De este análisis se intuye que, aunque haya un gran porcentaje de las empresas sociales nacionales en los territorios más poblados, que son comúnmente aquellos lugares donde existe un tejido empresarial significativo, hay un notorio arraigo de la Empresa Social en varias regiones menos pobladas.

Tabla. 1. Localización de las Empresas Sociales de España.

\begin{tabular}{|l|c|c|}
\hline \multicolumn{1}{|c|}{ Comunidad Autónoma } & Frecuencia & Porcentaje \\
\hline Andalucía & 516 & $19,95 \%$ \\
\hline Aragón & 85 & $3,29 \%$ \\
\hline Cantabria & 34 & $1,31 \%$ \\
\hline Castilla y León & 136 & $5,26 \%$ \\
\hline Castilla-La Mancha & 103 & $3,98 \%$ \\
\hline Cataluña & 345 & $13,34 \%$ \\
\hline Ceuta & 1 & $0,04 \%$ \\
\hline Comunidad de Madrid & 451 & $17,43 \%$ \\
\hline
\end{tabular}




\begin{tabular}{|l|c|c|} 
Región de Murcia & 83 & $3,21 \%$ \\
\hline Comunidad Valenciana & 190 & $7,34 \%$ \\
\hline Extremadura & 63 & $2,44 \%$ \\
\hline Galicia & 163 & $6,30 \%$ \\
\hline Islas Baleares & 39 & $1,51 \%$ \\
\hline Islas Canarias & 63 & $2,44 \%$ \\
\hline La Rioja & 12 & $0,46 \%$ \\
\hline Navarra & 42 & $1,62 \%$ \\
\hline País Vasco & 208 & $8,04 \%$ \\
\hline Principado de Asturias & 53 & $2,05 \%$ \\
\hline
\end{tabular}

Fuente: Elaboración propia a partir de Ashoka, Redcreactiva, Social Enterprise e Interès Social (2020).

De igual modo, los datos obtenidos señalan una consonancia con el Informe Global Entrepreneurship Monitor (2014) $)^{15}$, pues pone de relieve que Madrid es una región con un relevante porcentaje de población adulta involucrada en actividades de emprendimiento social. Por el contrario, La Rioja se encuentra a la cola del volumen de personas emprendedoras sociales.

\subsubsection{Ciclo de vida de la organización}

Se halla que la colectividad tiene más de 16 años de vida, pudiendo ser denominadas empresas de madurez avanzada (Gráfico 4 y 5). Por otra parte, el $58.45 \%$ de las Empresas Sociales españolas se crearon antes de la Gran Recesión (2007-2016).

Gráfico. 4. Ciclo de vida empresarial de las Empresas Sociales de España.

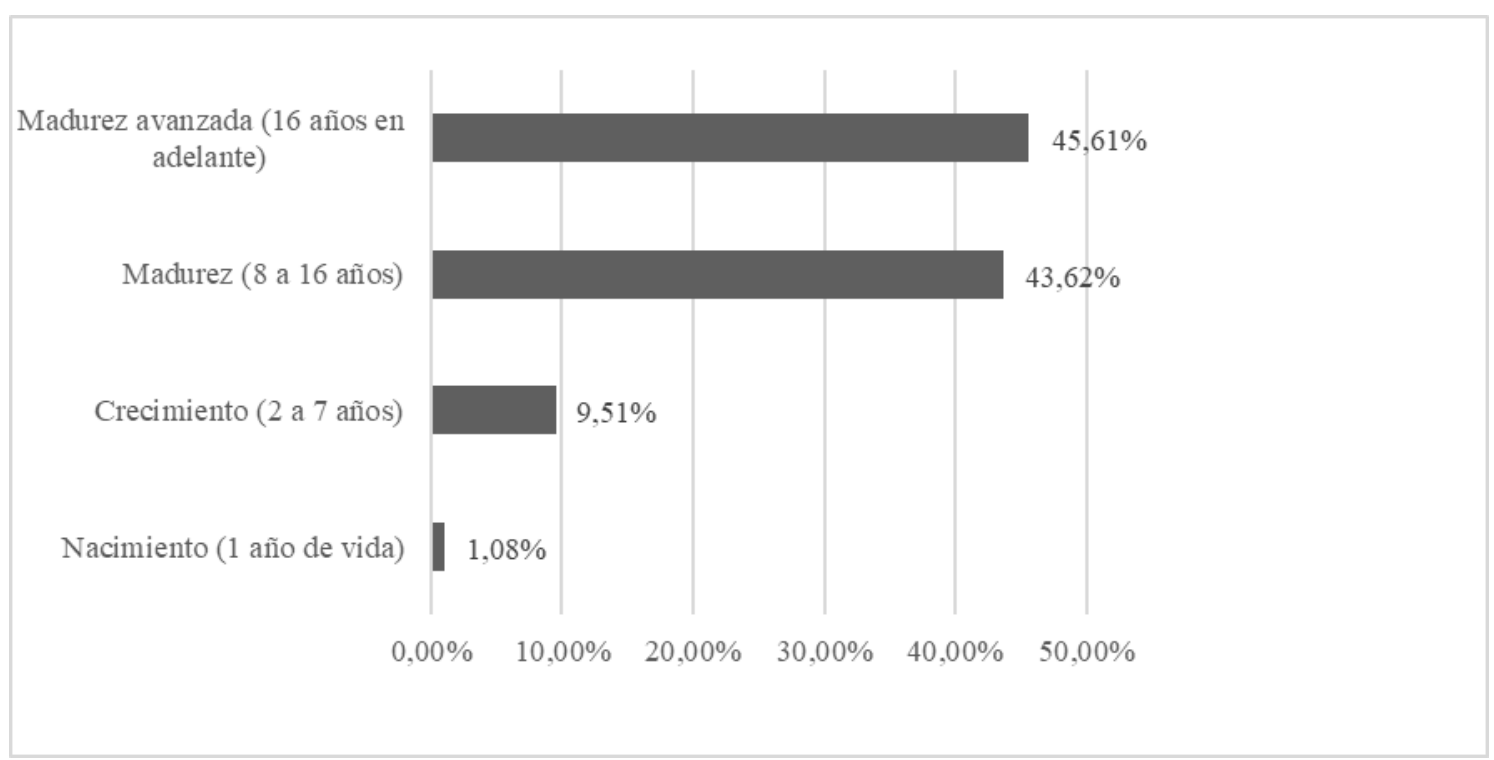

Fuente: Elaboración propia a partir de Ashoka, Redcreactiva, Social Enterprise e Interès Social (2020).

15 Global Entrepreneurship Monitor. 'Informe GEM España'. (Consultado 01.02.2021). http://www.gem-spain.com/wpcontent/uploads/2015/04/GEM es 2014.pdf. 
Gráfico. 5. Periodo de Constitución de las Empresas Sociales de España.

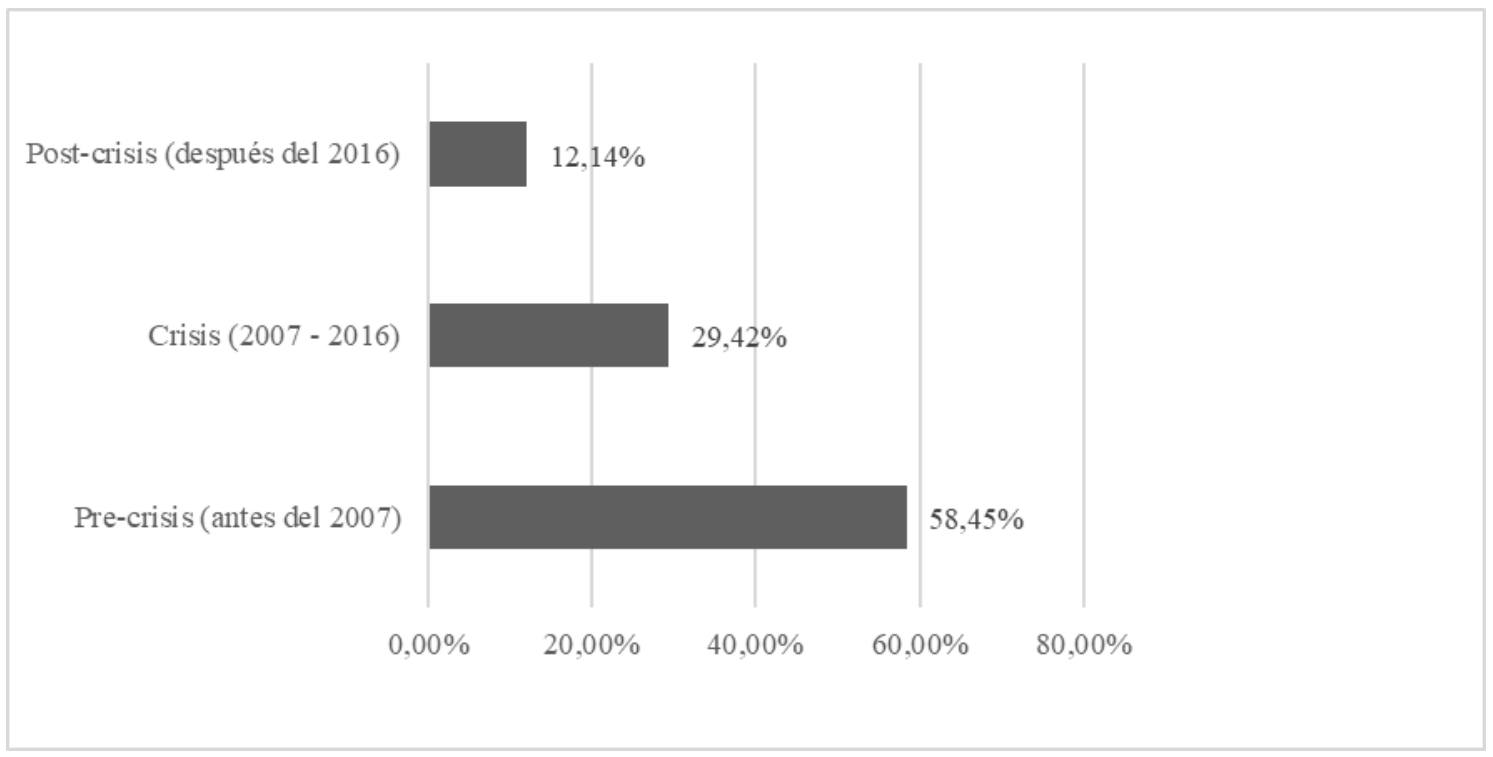

Fuente: Elaboración propia a partir de Ashoka, Redcreactiva, Social Enterprise e Interès Social (2020).

\subsubsection{Volumen de facturación}

El 34,38\% de las Empresas Sociales españolas factura más de 5 millones de euros anuales mientras que el resto, mayoritariamente, no factura más de 900.000 €/año (Gráfico 6).

Gráfico. 6. Volumen de facturación de las Empresas Sociales de España.

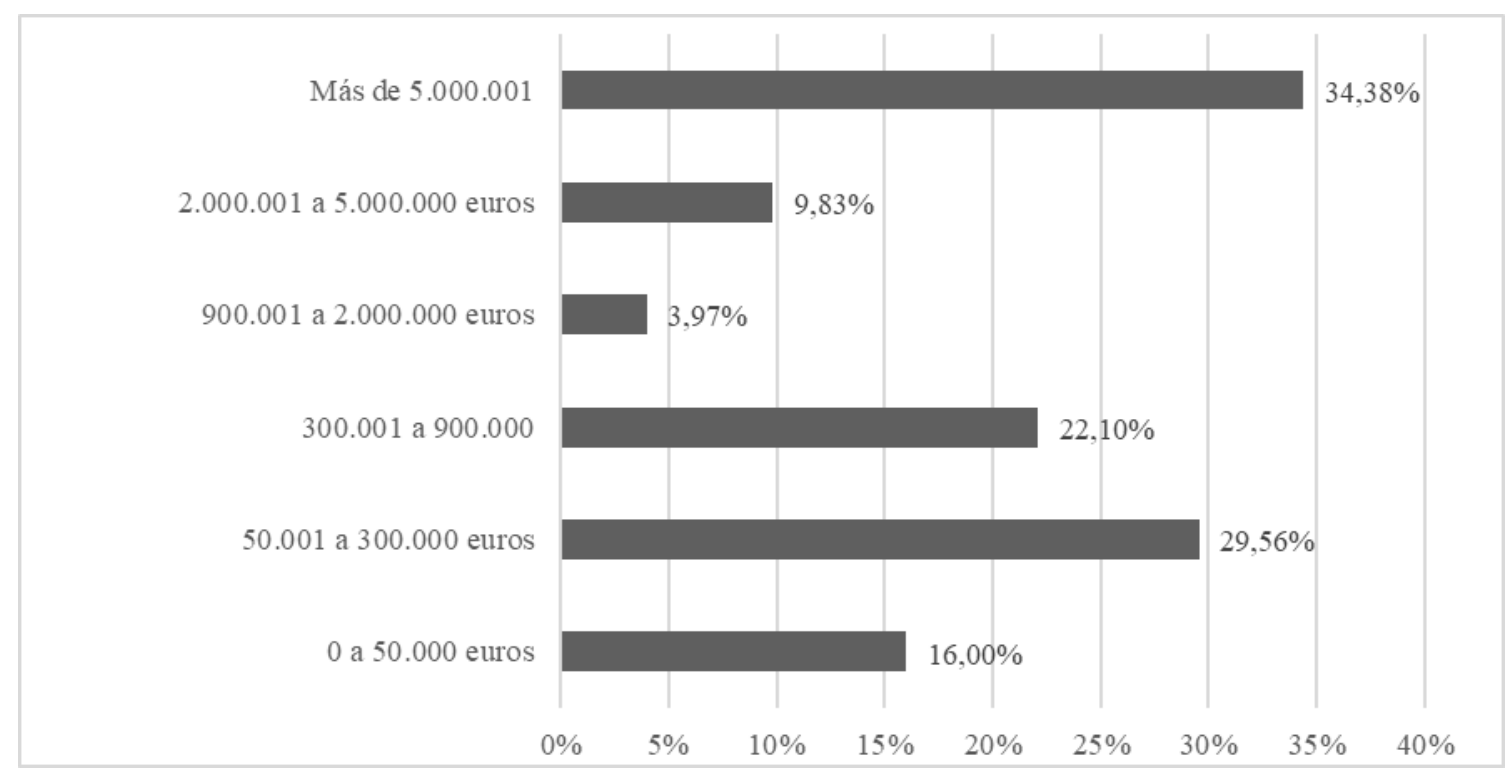

Fuente: Elaboración propia a partir de Ashoka, Redcreactiva, Social Enterprise e Interès Social (2020).

\subsubsection{Tamaño}

Desde el análisis del tamaño de las empresas según su número de personas empleadas, se observa que la mayoría de las empresas sociales son PYMES, empresas de 10 a 50 personas empleadas, seguidas de cerca por las microempresas ( 0 a 10 personas empleadas). La media de personas empleadas por empresa es de 168,10 , cifra que no es representativa de la realidad, pues la mediana es 24 con un rango intercuartílico de 541 personas (Tabla 2). 
Tabla. 2. Tamaño de las empresas según empleados/as.

\begin{tabular}{|l|c|}
\hline \multicolumn{1}{|c|}{ Tipología } & Porcentaje \\
\hline Microempresa (hasta 10 personas empleadas) & $35,76 \%$ \\
\hline Pequeña (de 10 a 50 personas empleadas) & $45,15 \%$ \\
\hline Mediana (de 50 a 250 personas empleadas) & $12,60 \%$ \\
\hline Grande (más de 250 personas empleadas) & $6,49 \%$ \\
\hline
\end{tabular}

Fuente: Elaboración propia a partir de Ashoka, Redcreactiva, Social Enterprise e Interès Social (2020).

En relación al número de personas empleadas, la evidencia responde como la Comisión Europea (2020:113), que España es una excepción a la norma, pues cuenta con varias grandes empresas sociales. La evidencia es que un 6,49\% de las empresas sociales españolas son grandes organizaciones.

Según las averiguaciones halladas, se puede detallar un arquetipo de la actual Empresa Social de España, tal como marca la Tabla 3.

Tabla. 3. El perfil de la Empresa Social (ES) activa en España.

\begin{tabular}{|l|l|}
\hline \multicolumn{1}{|c|}{ Variables } & \multicolumn{1}{c|}{ Derivaciones Principales } \\
\hline Servicios prestados & Sanidad $(48,72 \%)$ \\
\hline Sector productivo & Terciario $(57,15 \%)$ \\
\hline Forma Jurídica & Sociedad Limitada $(62,85 \%)$ \\
\hline Comunidad Autónoma & Andalucía $(19,95 \%)$ \\
\hline Periodo de Constitución & Pre-crisis antes de 2007 (58,45\%) \\
\hline Facturación & Más de 5 millones de euros $(34,38 \%)$ \\
\hline Tamaño & Pyme (10 a 50 personas trabajadoras) $(45,15 \%)$ \\
\hline
\end{tabular}

Fuente: Elaboración propia a partir de Ashoka, Redcreactiva, Social Enterprise e Interès Social (2020).

\subsection{El perfil de la Empresa Social activa en España según el ciclo de vida}

En base a las derivaciones anteriores, se hallan dos grupos en el ciclo de vida empresarial de las Empresas Sociales: el grupo de Nacimiento y Crecimiento (10,5\% de las empresas) y el grupo de Madurez y Madurez Avanzada (89,23\%). Ello marca la diferencia con las siguientes afirmaciones: la media de empresas sociales se encuentra en etapa temprana de desarrollo en España (GEM, 2014) ${ }^{16} \mathrm{y}$ las empresas sociales no son longevas (Arabadzhieva y Vutsova, 2021). En el reconocimiento de dos grupos, se hallan diferencias significativas en numerosas categorías (el * marca una diferencia porcentual significativa al 95\%). La Tabla 4 muestra una serie de derivaciones que plantean la importancia de seguir indagando en la materia.

Las empresas más jóvenes han reducido su participación en los sectores sustanciales, la actividad de sanidad (del 50,24\% al 35,93\%) y la manufactura (del 17,20\% al 3,70\%), mientras que han comenzado a operar en sectores poco frecuentados por empresas sociales como la construcción y el sector inmobiliario. De esta forma, y pese a que el sector terciario sigue siendo el sector más poblado por las empresas sociales, aquellas que se encuentran en fase de nacimiento y crecimiento han engordado su participación en el sector secundario. También se ha contemplado un cambio importante en la forma jurídica de las mismas. Por ejemplo, las empresas jóvenes se han constituido mayoritariamente como Cooperativas, reduciendo así la proporción de Sociedades Limitadas de nueva creación. Las regiones protagonistas se mantienen (Andalucía, Cataluña, Madrid, País Vasco y Galicia), aunque el ritmo de crecimiento ha diferido según comunidad. Por ejemplo, Madrid y País Vasco registraron un fuerte crecimiento de las sedes de empresas sociales. En cambio, Andalucía, Comunidad Valenciana y Cataluña, en menor medida, vieron como el número de empresas sociales de nueva creación se frenó dentro de sus límites.

Finalmente, con respecto a la facturación, se observa una tendencia atractiva. Quizás, debida a la configuración sectorial de sendos grupos. Las empresas maduras mantienen un equilibrio entre todos los rangos de facturación definidos, mientras que las empresas más jóvenes se encuentran en un escenario polarizado entre aquellas con grandes volúmenes de facturación y aquellas con pequeños volúmenes de facturación.

16 Global Entrepreneurship Monitor. 'Informe GEM España'. (Consultado 01.02.2021). http://www.gem-spain.com/wpcontent/uploads/2015/04/GEM es 2014.pdf. 
Tabla. 4. El perfil de la Empresa Social activa en España según el ciclo de vida empresarial.

\begin{tabular}{|c|c|c|c|}
\hline & & $\begin{array}{l}\text { Nacimiento y } \\
\text { crecimiento }\end{array}$ & $\begin{array}{c}\text { Madurez y } \\
\text { Madurez avanzada }\end{array}$ \\
\hline \multirow{17}{*}{ Servicios } & $\begin{array}{l}\text { Actividades profesionales / } \\
\text { científicas / técnicas }\end{array}$ & $3,33 \%$ & $3,09 \%$ \\
\hline & Administración y auxiliares & $12,96 \%$ & $13,67 \%$ \\
\hline & Agua y saneamiento & $0,00 \%$ & $0,13 \%$ \\
\hline & Arte y recreativas* & $1,48 \%$ & $0,35 \%$ \\
\hline & Comercio & $1,48 \%$ & $1,24 \%$ \\
\hline & Comunicación* & $3,33 \%$ & $6,57 \%$ \\
\hline & Construcción* & $32,59 \%$ & $2,78 \%$ \\
\hline & Educación & $1,11 \%$ & $0,57 \%$ \\
\hline & Energía & $0,37 \%$ & $0,18 \%$ \\
\hline & Finanzas & $0,00 \%$ & $1,15 \%$ \\
\hline & Ganadería & $0,37 \%$ & $0,04 \%$ \\
\hline & Hostelería & $0,00 \%$ & $0,49 \%$ \\
\hline & Inmobiliaria* & $1,11 \%$ & $0,22 \%$ \\
\hline & Manufacturas* & $3,70 \%$ & $17,20 \%$ \\
\hline & Otros & $0,00 \%$ & $0,79 \%$ \\
\hline & Sanidad* & $35,93 \%$ & $50,24 \%$ \\
\hline & Transporte y almacenamiento & $2,22 \%$ & $1,28 \%$ \\
\hline \multirow{3}{*}{ Sector Productivo } & Primario & $0,37 \%$ & $0,048 \%$ \\
\hline & Secundario* & $36,67 \%$ & $12,82 \%$ \\
\hline & Terciario* & $62,96 \%$ & $87,11 \%$ \\
\hline \multirow{7}{*}{ Forma Jurídica } & Asociaciones & $0 \%$ & $0,14 \%$ \\
\hline & Sociedad Limitada* & $55,47 \%$ & $70,82 \%$ \\
\hline & Sociedad Anónima & $0 \%$ & $0,48 \%$ \\
\hline & Sociedad Laboral* & $2,55 \%$ & $13,91 \%$ \\
\hline & Fundaciones & $0,36 \%$ & $0,34 \%$ \\
\hline & Cooperativas* & $41,24 \%$ & $13,24 \%$ \\
\hline & $\begin{array}{l}\text { Entidad de Previsión Social } \\
\text { Voluntaria }\end{array}$ & $0,36 \%$ & $1,06 \%$ \\
\hline \multirow{18}{*}{ Localización } & Andalucía* & $11,68 \%$ & $19,08 \%$ \\
\hline & Aragón & $3,28 \%$ & $3,09 \%$ \\
\hline & Cantabria* & $0 \%$ & $1,49 \%$ \\
\hline & Castilla y León & $4,37 \%$ & $4,92 \%$ \\
\hline & Castilla-La Mancha & $1,82 \%$ & $3,57 \%$ \\
\hline & Cataluña & $12,04 \%$ & $14,3 \%$ \\
\hline & Ceuta & $0 \%$ & $0,04 \%$ \\
\hline & Comunidad de Madrid* & $32,84 \%$ & $16,86 \%$ \\
\hline & Comunidad de Murcia & $2,91 \%$ & $3,23 \%$ \\
\hline & Comunidad Valenciana* & $4,37 \%$ & $4,97 \%$ \\
\hline & Extremadura & $0,72 \%$ & $2,41 \%$ \\
\hline & Galicia & $5,47 \%$ & $6,81 \%$ \\
\hline & Islas Baleares & $1,09 \%$ & $1,04 \%$ \\
\hline & Islas Canarias & $2,19 \%$ & $2,70 \%$ \\
\hline & La Rioja & $0 \%$ & $0,5 \%$ \\
\hline & Navarra & $1,09 \%$ & $1,79 \%$ \\
\hline & País Vasco* & $14,96 \%$ & $7,34 \%$ \\
\hline & Principado de Asturias & $1,09 \%$ & $2,27 \%$ \\
\hline \multirow{6}{*}{$\begin{array}{l}\text { Volumen } \\
\text { facturación }\end{array}$} & 0 a 50.000 euros* & $0,37 \%$ & $0,15 \%$ \\
\hline & 50.001 a 300.000 euros & $33,96 \%$ & $32,17 \%$ \\
\hline & 300.001 a $900.000 *$ & $7,54 \%$ & $25,38 \%$ \\
\hline & 900.001 a 2.000 .000 euros & $2,26 \%$ & $3,31 \%$ \\
\hline & 2.000 .001 a 5.000 .000 euros & $9,43 \%$ & $10,05 \%$ \\
\hline & Más de 5.000.001* & $46,41 \%$ & $28,90 \%$ \\
\hline
\end{tabular}

Fuente: Elaboración propia a partir de Ashoka, Redcreactiva, Social Enterprise e Interès Social (2020). 


\section{Conclusiones}

El tratamiento de la muestra de 2.587 empresas sociales registradas permite delimitar el perfil vigente de la Empresa Social en España. La Empresa Social española es una Sociedad Limitada PYME que se dedica a actividades sanitarias, principalmente. Al igual que señaló la Comisión Europea (2020:84), "las empresas sociales participan en todos los campos de actividad, especialmente en los servicios sanitarios y en el de inserción laboral". Por tanto, la segunda hipótesis se verifica (las Empresas Sociales españolas ostentan unas características propias).

Con respecto a la primera hipótesis (las Empresas Sociales españolas se crean en periodo de crisis económica como durante la Gran Recesión, 2007-2016), un significativo 29,42\% de las empresas tratadas se fundó durante la crisis económica de 2008. En este sentido, el análisis del ciclo de vida empresarial nos indica que un 43,62\% de las empresas sociales analizadas nació durante el periodo 2004-2012, coincidiendo con el ciclo económico completo que se dio desde mediados de los 2000 hasta mediados de los años 2010. Ello sugiere que las empresas sociales generan estabilidad en épocas de crisis. De igual modo, se cumple la tercera hipótesis (las Empresas Sociales residen donde la Economía Social nacional), pues las Empresas Sociales residen en Andalucía y la Comunidad de Madrid, regiones protagonistas de la Economía Social nacional. Se evidencia que las Empresas Sociales españolas habitan en las regiones más pobladas, aunque cuentan con un arraigo en otras regiones como Aragón y País Vasco, también con tradición en la Economía Social.

Las implicaciones de la investigación pasan por contribuir tanto al reciente debate sobre el proceso de crecimiento de las empresas sociales como a aquellas investigaciones que señalan que la Empresa Social en España no supone una excepción a las existentes en el contexto internacional (Solórzano-García et al., 2018). Sin duda, debe señalarse que el análisis descriptivo presentado se suma a otras primeras aproximaciones sobre la comprensión de la Empresa Social paradigmática en España. Pese a que la muestra de empresas es amplia y procede de diversas fuentes (base de datos digitales), se deben señalar las limitaciones de la investigación que son de índole temporal y sobre la variedad de recursos consultados. Las futuras investigaciones deben profundizar la revisión de la literatura a partir de su extensión y el detalle del ciclo de vida de la organización, desmenuzar el funcionamiento organizacional e indagar la tendencia tipológica. A nivel metodológico, abordar una temporalidad más amplia a partir de recabar datos de varios años e incrementar los métodos de captación de datos, las variables observadas y las técnicas cuantitativas tratadas. Se podría medir el impacto social a través de la tasa de recuperación de inversiones sociales - Social Return on Investment (SROI) - (Díaz-Foncea, Marcuello y Marcuello-Servós, 2012:187). Asimismo, realizar un estudio comparado sobre los efectos de la reciente crisis económica del emprendimiento en empresas de Economía Social (Cantarero, González-Loureiro y Puig, 2017) y Empresas Sociales en España. Otras propuestas serían: determinar el papel de las empresas sociales sociosanitarias durante y pos la COVID-19; conocer a las empresas creadas durante el periodo de la Gran Recesión durante la esperada recuperación COVID-19; plantear algunas indagaciones pormenorizadas sobre las empresas sociales españolas activas, pues "académicamente es interesante analizar experiencias reales en el ámbito de las cooperativas de iniciativa social" (Burgo, 2014:78); al igual que comprobar la relación de las empresas sociales con los Objetivos de Desarrollo Sostenible 2030 (ODS).

\section{Referencias bibliográfícas}

Altzelai Uliondo, I. (2020) Un marco jurídico para la Empresa Social en la Unión Europea. CIRIEC-España, Revista Jurídica de Economía Social y Cooperativa, No 37, pp. 105-140. DOI: 10.7203/CIRIEC-JUR.37.17845.

Álvarez Vega, M.I. (1999) Las Empresas de inserción social como nueva forma de organización empresarial. Especial referencia a su régimen jurídico. CIRIEC-España, Revista de Economía Pública, Social y Cooperativa, № 31, pp. 47-84.

Arabadzhieva, M.; Vutsova, A. (2021) Social enterprises' ecosystem - status quo and its auspicious development. REVESCO. Revista de Estudios Cooperativos, vol. 137, e71864. https://dx.doi.org/10.5209/reve.71864.

Aretxabala M.E., \& Caro, A. (2013) Las empresas de inserción vascas y sus entidades promotoras como ejemplo de buenas prácticas en la integración sociolaboral del colectivo de inmigrantes más vulnerable. Revista Vasca de Economía Social, $\mathrm{N}^{\circ}$ 10, pp. 149-188.

Argudo Périz, J.L. (2011) Las cooperativas sin ánimo de lucro: ¿vuelta a los orígenes o respuesta a nuevas necesidades sociales? Revista Vasca de Economía Social, № 3, pp. 179-201.

Berbegal-Mirabent, J., Mas-Machuca, M., \& Guix, P. (2021) Impact of mission statement components on social enterprises' performance. Review of Managerial Science, $\mathrm{N}^{\circ}$ 15, pp. 705-724. https://doi.org/10.1007/s11846-019$\underline{00355-2}$. 
Bretos, I., Errasti, A., \& Marcuello, C. (2020) Is there life after degeneration? The organizational life cycle of cooperatives under a 'grow-or-die'dichotomy. Annals of Public and Cooperative Economics, No 91, pp. $435-458$. https://doi.org/10.1111/apce.12258.

Bretos, I.; Díaz-Foncea, M., \& Marcuello, C. (2020) La cooperativa de iniciativa social: un modelo de Empresa Social en España. REVESCO. Revista de Estudios Cooperativos, No135, pp. 1-19. DOI: https://doi.org/10.5209/reve.69186.

Burgo García, U. (2014) El movimiento cooperativo de las ikastolas: su revisión conceptual desde la perspectiva de las empresas sociales. Revista Vasca de Economía Social, Nº10, pp. 71-96.

Cantarero, S., González-Loureiro, M. y Puig, F. (2017) Efectos de la crisis económica sobre el emprendimiento en empresas de economía social en España: un análisis espacial. REVESCO. Revista de Estudios Cooperativos, Tercer Cuatrimestre, $\mathrm{N}^{\circ} 125$, pp. 24-47. DOI: 10.5209/REVE.56133.

Caro-González, F.J., Pérez-Suárez, M., \& Sánchez-Torné, I. (2019) Características de las Empresas Sociales Periodísticas. CIRIEC-España, Revista de Economía Pública, Social y Cooperativa, No 96, pp. 121-154. https://doi.org/10.7203/CIRIEC-E.96.12723.

COMISIÓN EUROPEA (2020) Las empresas sociales y sus ecosistemas en Europa. Informe de síntesis comparativo. Disponible en https://ec.europa.eu/social/main.jsp?catId=738\&langId=en\&pubId=8274.

Coque, J., \& Pérez, E. (2000) Cómo gestionar la creación de empresas de inserción social: una perspectiva de participación dinámica, en Parras, M. (Ed.) Inteligencia empresarial. La gestión del conocimiento en la empresa, $\mathrm{N}^{\circ}$ 2, pp. 333-43. Jaén: Instituto de Estudios Giennenses.

Defourny, J., \& Nyssens, M. (2014) Social co-operatives: When social Enterprises meet the co-operative tradition. Journal of Entrepreneurial and Organizational Diversity, Vol. 2, $\mathrm{N}^{\mathrm{o}}$ 2, pp.11-33. Disponible en https://papers.ssrn.com/sol3/papers.cfm?abstract_id=2437884.

Díaz-Foncea, M. (Coord.) (2017) Social Enterprise in Spain: A Diversity of Roots and a Proposal of Models. ICSEM Working Papers, No 29. Disponible en https://zaguan.unizar.es/record/70184/.

Díaz-Foncea, M., \& Marcuello, C. (2014) Las empresas sociales en España: concepto y características. Revista Vasca de Economía Social, № 8, pp. 143-164.

Díaz-Foncea, M., Marcuello, C., \& Marcuello-Servós, CH. (2012) Empresas sociales y evaluación del impacto social. CIRIEC-España, Revista de Economía Pública, Social y Cooperativa, No 75, pp. 181-198. Disponible en https://www.redalyc.org/pdf/174/17425798010.pdf.

FAEDEI (2003) Identificación y diagnóstico integral de las Empresas de Inserción en España. Madrid: Editorial Popular.

Fajardo García, G. (2014) El concepto legal de Economía Social y la Empresa Social. Revista Vasca de Economía Social, $\mathrm{N}^{\mathrm{o}} 8$, pp. 63-84.

Fisac-García, R., \& Moreno-Romero, A.M. (2015) La Empresa Social: marco conceptual, contexto e información. Documentos AECA, Serie Responsabilidad Social Corporativa, $\mathrm{N}^{\mathrm{o}}$ 9, pp. 1-94. Disponible en http://www.aeca1.org/revistaeca/revista112/112.pdf.

Fisac-García, R., Moreno-Romero, A.M., Mataix, C., \& Palacios, M. (2011) La Empresa Social: revisión de conceptos y modelo para el análisis organizativo. Revista Española del Tercer Sector, pp. 41-66. Disponible en http://oa.upm.es/12404/.

García Calavia, M.A. (2020) Las empresas de inserción en España en 2019. CIRIEC-España, Revista Jurídica de Economía Social y Cooperativa, No 36, pp. 131-152. DOI: 10.7203/CIRIEC-JUR.36.17307.

García, A., \& Esteve, M. (2007) Las Empresas de Inserción en España: normativa y características. CIRIEC-España, Revista de Economía Pública, Social y Cooperativa, No 59, pp. 153-78.

García, M. A., Herrero, M., \& Martínez, I. (2020) Empresas sociales, Empresas de Inserción y Centros Especiales de Empleo. In Manual de Economía Social, pp. 369-396. Tirant lo Blanch.

Laville, J.L. (2015) Asociarse para el bien común: Tercer Sector, Economía Social y Economía Solidaria. Barcelona: Icaria.

López, M.C., Maside, J. M. y Torrelles, J. (2019) Análisis económico y social de los centros especiales de empleo: un estudio en Galicia. REVESCO. Revista de Estudios Cooperativos, Tercer Cuatrimestre, No 132, pp. 169-194. https://doi.org/10.5209/reve.64305.

López-Aranguren, L.M. (2002) Las empresas de inserción en España: un marco de aprendizaje para la inserción laboral. Madrid: Consejo Económico y Social.

Marcuello, C. (2007) Empresas de inserción: puentes de inclusión en el mercado laboral. CIRIEC-España, Revista de Economía Pública, Social y Cooperativa, No 59, pp. 5-6.

Marcuello, C., \& Marcuello-Servós, CH. (2010) Las Empresas de Inserción, en Mozón, E. \& Monzón-Campos, J.L. (Eds.), La Economía Social en España en el año 2008. Ámbito, magnitudes, actividades y tendencias, pp. 417-33. CIRIEC-España, Valencia.

Marcuello, C., Bellostas, A., \& Marcuello-Servós, CH. (2008) Informe sobre las Empresas de Inserción en España, Valencia: CIRIEC-España.

Martínez-Rueda, N., Aróstegui, I. \& Galarreta, J. (2018) Factores organizacionales que inciden en la mejora de la empleabilidad de las Empresas de Inserción. CIRIEC-España, Revista de Economía Pública, Social y Cooperativa, No 94, pp. 123-154. DOI: 10.7203/CIRIEC-E.94.12697.

Melián, A., Campos, V. y Sanchis, J.R. (2011) Emprendimiento social y empresas de inserción en España. Aplicación del método Delphi para la determinación del perfil del emprendedor y las empresas sociales creadas por 
emprendedores. REVESCO. Revista de Estudios Cooperativos, Tercer Cuatrimestre, № 106, pp. 150-172. DOI: 10.5209/rev_REVE.2011.v106.37377.

Millán, J.C. (2006) Empresas de Inserción. Santiago de Compostela: Instituto Gallego de Iniciativas Sociales y Sanitarias.

Monzón-Campos, J., \& Chaves-Ávila, R. (2012) Evolución reciente de la Economía Social en la Unión Europea. Bélgica: CIRIEC-International. CES/CSS/12/2016/23406. Disponible en https://www.eesc.europa.eu/sites/default/files/files/qe-04-17-875-es-n.pdf.

Nogales-Muriel, R. (2017) La Empresa Social en Europa y España: evolución, relevancia y desafíos. Revista Tercer Sector, pp. 117-140. Disponible en https://cendocps.carm.es/documentacion/2017_RevistaEspa\%C3\%B1olaTercerSector.\%20N.35.pdf\#page=117.

Paniagua Zurera, M. (2013) Las empresas de inserción sociolaboral y las cláusulas sociales en la contratación pública: dos instrumentos necesarios para la innovación social hacia el desarrollo. REVESCO. Revista de Estudios Cooperativos, N ${ }^{\circ} 112$, pp. 176-212. http://dx.doi.org/10.5209/rev_REVE.2013.v112.43066.

Pfeilstetter, R.; Gómez-Carrasco, I. (2020) Local meanings of social enterprise. A historical-particularist view on hybridity of organizations. REVESCO. Revista de Estudios Cooperativos, vol. 134, e69162. https://dx.doi.org/10.5209/REVE.69162.

Puig i Olle, A. (1998) Las Empresas de Inserción. El papel de las fundaciones y las asociaciones en la creación de las empresas. REVESCO. Revista de Estudios Cooperativos, $\mathrm{N}^{\circ}$ 65, pp. 69-71.

Quintão, C. (2007) Empresas de Inserción y Empresas Sociales en Europa. CIRIEC-España, Revista de Economía Pública, Social y Cooperativa, No 59, pp. 5-6.

Retolaza, J.L., San-José, L. y ARAUJO, A. (2014) The Efficiency as a Challenge of Work Insertion Social Enterprises. REVESCO. Revista de Estudios Cooperativos, Segundo Cuatrimestre, No 115, pp. $159-185$. http://dx.doi.org/10.5209/rev_REVE.2014.v115.45276.

Retolaza, J.L., San-José, L., \& Pruñonosa, J.T. (2013) Las empresas de inserción en España: ¿una oportunidad perdida? Revista Vasca de Economía Social, № 10, pp. 97-123.

Rodríguez, A. (2015) Algunas consideraciones sobre el ánimo de lucro en las cooperativas de iniciativa social. Análisis de su relación con los principios cooperativos. CIRIEC-España, Revista Jurídica de Economía Social y Cooperativa, $\mathrm{N}^{\circ}$ 26, pp. 1-46. Disponible en http://ciriec-revistajuridica.es/wp-content/uploads/026-007.pdf.

Rojo Giménez, C. (2000) Empresas de Inserción en Castilla y León. CIRIEC-España, Revista de Economía Pública, Social y Cooperativa, $\mathrm{N}^{\mathrm{o}} 36$, pp. 97-115.

Salinas, F., \& Rubio, M.J. (2001) Tendencias en la evolución de las organizaciones no lucrativas hacia la Empresa Social. CIRIEC-España, Revista de Economía Pública, Social y Cooperativa, No 37, pp. 79-116. Disponible en https://www.redalyc.org/pdf/174/17403705.pdf.

Sánchez Pachón, L.Á. (2020) Los Centros Especiales de Empleo: configuración legal e incidencia y valoración de las últimas actuaciones normativas. CIRIEC-España, Revista Jurídica de Economía Social y Cooperativa, No 36, pp. 55-91. DOI: 10.7203/CIRIEC-JUR.36.16989.

Sánchez-Torné, I., \& Pérez-Suárez, M. (2020) A State Diagnostic of the Social Enterprise in Spain. GIZAEKOA-Revista Vasca de Economía Social, № 17, pp. 7-34. https://doi.org/10.1387/gizaekoa.22225.

Solórzano-García, M., Guzmán-Alfonso, C., Savall-Morera, T., \& Villajos-Girona, E. (2018) La identidad de la Empresa Social en España: análisis desde cuatro realidades socioeconómicas. CIRIEC-España, Revista de Economía Pública, Social y Cooperativa, No 92, pp. 155-182. https://doi.org/10.7203/CIRIEC-E.92.9236.

Spear, R., Cornforth, C., \& Aiken, M. (2009) The governance challenges of social enterprises: evidence from a UK empirical study. Annals of Public and Cooperative Economics, $\mathrm{N}^{\mathrm{o}} \quad 80(2)$, pp. $247-273$. https://doi.org/10.1111/j.1467-8292.2009.00386.x.

Torres Ortega, J.A. (2017) Ecosistemas para el emprendimiento: Características del concepto y su aplicación a la Empresa Social. Revista Vasca de Economía Social, No 14, pp. 61-76. DOI: 10.1387/reves.18004. 